\title{
Sistema y mundo de la vida en la acción comunicativa
}

\author{
Marco Antonio Millán Campuzano* \\ Universidad Autónoma Metropolitana, Unidad Cuajimalpa
}

El presente artículo sintetiza una conocida posición teórica de Jürgen Habermas con el objeto de esclarecer datos y materiales fértiles a una discusión sobre una posible Ciencia de la Comunicación.

This article synthetizes a know theoric position of Jürgen Habermas with the unique objective to elucidate facts and fertile materials on a discussion about the possibility of a Communication Science.

\section{INTRODUCCIÓN}

El artículo recoge la polémica que Habermas establece con el idealismo hermenéutico, de herencia fenomenológica, de la sociología comprensiva. Es en la Teoría Acción Comunicativa donde se establece dicha discusión que marca las limitaciones que, vista desde la ética discursiva, tiene la sociología de Shütz en relación a la conformación del concepto de intersubjetividad en el mundo-de-lavida. Mostrar esta discusión no es baladí para los estudios de la comunicación, pues representa parte de una polémica aún vigente acerca de los conceptos de sujeto e intersubjetividad como propicios al terreno teórico de la Comunicación como mundo de la vida.

* Lic. en Periodismo y Comunicación Colectiva, Maestro en Filosofía de la Ciencia y candidadato a Doctor en Filosofía de la Ciencia. Profesor de la Academia de Filosofía de la UACM, Profesor investigador invitado de la UAM-Cuajimalpa y profesor de asignatura de la Universidad Iberoamericana. 
¿Dónde ocurre la dinámica de la intersubjetividad? ¿Dónde se da la acción orientada a los acuerdos? ¿En qué lugar ocurre la acción comunicativa? ¿En qué sitio la razón práctica? ¿Basta con suponer la necesidad de la reflexión acerca de la intersubjetividad para alcanzar claridad en el ámbito comunicativo del mundo-de-la-vida?

Habermas sabe que en su proyecto teórico la racionalización requiere no sólo de modelos, categorías y conceptos, sino también de explicitar qué tiene que hacer todo aquello en una cultura concreta, en la vida social y personal, en una palabra: en un mundo de la vida dado.

Sí, Habermas probablemente lo sabe (¿tenemos alguna razón para dudar de ello? Ya veremos) y por eso habla del mundo de la vida. Del suyo, de éste o de aquel, quizá no importe cuál en específico, pues la "universalidad" es de amplia ambición en su proyecto teórico. Algo inevitable sale a flote como perspectiva de su enfoque: la modernidad y el proyecto racional que nuestro autor sustenta. Habermas no es un necio en tiempos "posmodernos", aunque lo parece. Remar contra la corriente de los pos y la marea alta de la desconstrucción es cosa riesgosa y, a la vez, necesaria.

Sistema y mundo de la vida es el tema de fondo, y es la reflexión polémica que se abre con estos temas. Sistemas sociales, sistemas dinámicos, totalitarios, burocráticos, dominados, periféricos, ilusorios, increíbles, carniceros y más. Mundos vitales complejos, mundos de vida efímera, inmediatos, incrédulos, donde uno se mueve, bosteza, camina, duerme, charla, explica y da razones, sin razones y comezones. Sistema y mundo de la vida. Este es el tema de fondo. Entraremos a él.

Hay una precisión que conviene decir desde ahora. La ofrece Richard Bernstein: "la perspectiva teórica de Habermas ayuda también a iluminar nuestras perspectivas futuras, -no en el sentido de predecir el futuro, sino más bien subrayando conceptualmente la necesidad de fomentar la racionalización sistemática y la racionalización comunicativa del mundo vital' (Bernstein 1991, p. 125). Se observa que hay una relación entre el mundo de la vida cotidiano, real y el sistema como instancia sobre la que se mueven los miembros de una comunidad, ambos guiados por la racionalidad.

Y es que a las teorías sistémicas (vgr. Parsons, Luhmann) se les escapa el saber intuitivo del mundo de la vida y de sus miembros, y la razón de ello es que las sociedades modernas son, cada vez, más complejas y por tanto, más 
difíciles de abarcar en su plena dimensión, es decir, no es lo mismo plantear una sociedad como sistema, a través de modelos abstractos, que entenderla como un mundo de la vida dinámico. Habermas así lo observa y adapta el concepto Husserliano de "mundo de la vida" a su teoría de la acción comunicativa, vía pragmática del lenguaje (no formal sino orientada al acuerdo) sin descuidar a la sociedad como sistema.

Así hay, pues, por lo menos, dos formas inmediatas de conceptuar a la sociedad:

a) como mundo de la vida

b) como Sistema

La propuesta de nuestro autor será la de entender simultáneamente ambos planteamientos, para lo cual desarrollará 5 pasos encaminados a esclarecer sus ideas: 1) Relacionará el mundo de la vida con aquellos tres momentos (o mundos) en los que los sujetos se ven involucrados, a "fin" de orientar acuerdos, 2) Desarrollará el mundo de la vida como contexto de la acción comunicativa, sirviéndose del análisis fenomenológico (Husserl) y vinculando este con el concepto de conciencia colectiva (Durkheim), 3) Hará notar cómo los conceptos de mundo de la vida "usuales" en sociología, que parten de representaciones cotidianas, sólo son útiles a la exposición narrativa, 4) Analizará las condiciones necesarias para una racionalización del mundo de la vida, partiendo de las funciones que la acción comunicativa desempeña para mantener un mundo de la vida estructuralmente diferenciado y 5) Propondrá atender simultáneamente a la sociedad como sistema y como mundo de la vida.

Así, en este mismo orden, expondremos lo formulado por Habermas. La exposición tratará de ser fiel (con "transcripciones transfiguradas" concientes) y acaso clarificadora (cosa difícil pues Habermas es claro). Al final, a manera de conclusión no cerrada, se plantearán tres cuestionamientos ambiguos, pero no irrelevantes.

El concepto de mundo de la vida estará vinculado al de acción comunicativa. El mundo de la vida es el lugar en donde agentes comunicativos se mueven ya siempre (es decir que tiene una carácter trascendental).

Dentro del mundo de la vida Habermas distingue tres relaciones (o submundos) que los sujetos que participan en acciones orientadas a acuerdos ponen como base (lo quieran o no) de una situación concreta.

Los sujetos se relacionan con:

- Algo en el mundo objetivo ("como totalidad de las entidades sobre las que son posibles enunciados verdaderos") 
- Algo en el mundo social ("como totalidad de las relaciones interpersonales legitimamente reguladas")

- Algo en el mundo subjetivo ("como totalidad de las propias vivencias a las que cada cual tiene un acceso privilegiado y que el hablante puede manifestar verazmente ante un público") ( Habermas 2001, p.171)

En una acción comunicativa habrá un proceso cooperativo de interpretación de estos tres referentes. Los participantes se verán involucrados simultáneamente en los tres "mundos", aunque en alguna situación específica se manifieste, con mayor énfasis, sólo uno de los tres componentes.

En el mundo de la vida se dan situaciones de cotidianidad, en las que se ponen a transitar, implícitamente, las tres relaciones arriba mencionadas. Planteemos una situación a manera de ejemplo: en un aula de clases ocurre que el profesor pide a un alumno que busque un proyector de acetatos para la clase que empezará dentro de 5 minutos. Tiempo considerable para que se cumpla la petición hecha por el profesor al alumno y la clase comience a tiempo. Aquí se dan por hecho algunas cosas: que los implicados tienen clara la situación; que hay una hora para que comience la clase (tema); que el maestro pueda emitir la petición sin réplica (plan); y que el alumno es casi imposible que se niegue (status).

En este ejemplo se destacaría el mundo social (sólo se destacaría) de los otros dos, en el hecho de que en la situación prevalece un marco normativo (institucional si se quiere) en el que se puede exigir que otro haga algo. El mundo objetivo (el proyector, el salón), y el mundo subjetivo (apreciaciones del alumno hacia la clase y/o profesor, aburrimiento, disponibilidad) quedarían inmersos en el mundo social. No excluidos, sólo inmersos y actuando ya siempre, como trasfondo de una emisión comunicativa orientada al logro de un acuerdo y a la satisfacción de la necesidad inmediata del acuerdo mismo. Esto último queda de manifiesto cuando el tema se desplaza (la clase, en el ejemplo mencionado) y con él, el horizonte de la situación (si la utilización del aparato proyector de acetatos se suspende por un corte de energía eléctrica) y con éste, el "fragmento" de mundo de la vida relevante para ese momento.

Una situación dada se verá modificada cuando el horizonte se ve, a su vez, desplazado por alguna otra situación acaecida (el maestro olvidó los acetatos; los acetatos estaban borrosos; el alumno no encontró el proyector; el maestro fue avisado del fallecimiento de la abuelita de Juancito Pérez, etc.) Y claro, como ya es sabido en el horizonte habermasiano, dentro de estos casos de acción 
comunicativa de un mundo de la vida, es preciso asentir las pretensiones de validez, pues de no hacerlo, no habrá acuerdos (razón y sentido último de la acción comunicativa en el terreno de la intersubjetividad).

Ahora bien, para que alguna situación de acción se torne relevante es necesario que ésta, en tanto horizonte que se desplaza en el mundo de la vida (trasfondo siempre presente), pierda trivialidad y solidez incuestionada al momento en que es sabida y problematizada como hecho, como contenido de una norma y/o como contenido de una vivencia.

En otras palabras, se podría decir que el mundo de la vida actúa como plataforma siempre presente en la que los sujetos se mueven intuitivamente, sintiendo a aquel como depositario de autoevidencia (que los hace ser y ser en su mundo sin preguntas) o convicción incuestionada, pero cuando se da una situación relevante, las convicciones y autoevidencias son movilizadas en forma de saber (donde existe consenso) que es susceptible de ser problematizado. Una situación a-problemática se torna relevante gracias a los plexos semánticos que median entre un horizonte de connotaciones semánticas, y un contexto inmediato, dentro de una emisión comunicativa.

Ahora podríamos preguntarnos, ¿qué es lo que provee a los participantes en la comunicación de convicciones que son aproblemáticas y que ellos suponen garantizadas? La respuesta que ofrece nuestro autor es clara si comenzamos por separar los mundos formales (objetivo, subjetivo y, social) y el lenguaje y la cultura como tales, pues a éstos se les cumple un papel "trascendental" (ojo: estamos ante un neoapelianismo de corte Kantiano), dentro de todo aquello que es susceptible ser problematizado en una situación. La razón de la tal separación se entiende cuando se da por hecho que el lenguaje y la cultura son partes constitutivas del mundo de la vida, mientras que los conceptos formales sólo sirven para armar categorías útiles para clasificar situaciones dadas. Además, cuando se ejecuta un acto de habla orientado al acuerdo, los que participan en éste se mueven, (ya siempre) dentro de su lenguaje. El lenguaje no puede ser problematizado como algo objetivo, social o subjetivo, pues es el vehículo mismo (aunque no simple instrumento de los "mundos") sobre el que transitan los acuerdos y que actúa a espaldas de los involucrados en una actitud performativa. La cultura actúa de un modo semejante, pues a través de lenguajes se trasmiten los patrones culturales que le son familiares a la comunidad (tradiciones, vestido, creencias, etc.) Toda situación emerge de un mundo de la vida, que culturalmente nos es familiar y por tanto, nunca será totalmente desconocida. 
Ahora es clara la respuesta: lo que garantiza las convicciones la a-prolematicidad es el trasfondo (mundo de la vida) lingüístico-cultural en el que una comunidad se desarrolla y que siente existir, ya siempre. Pero cuando algún plexo es movido a una situación que requiera de un acuerdo, se problematiza y se canaliza, por así decirlo, a algo objetivo, subjetivo o social, que será abordado desde una racionalidad motivada por argumentos.

Finalmente citaremos a Habermas:

“...al mundo de la vida le es constitutivo el acuerdo como tal, mientras que los conceptos formales de mundo establecen un sistema de referencia para aquello sobre lo que el acuerdo es posible: hablante y oyente se entienden desde, y a partir de, el mundo de la vida que les es común sobre algo en el mundo objetivo, en el mundo social y en el mundo subjetivo" (Habermas 2001, p.192).

Habermas establece una comparación entre el concepto fenomenológico de mundo de la vida y el propio, de teoría de la acción comunicativa. Su "sparring" será Shütz habilitado por Luckmann. El referee, el lector mismo. Hacemos la presentación en sus respectivas esquinas.

ACCIÓN COMUNICATIVA

Destaca dos aspectos, en tanto formas de habérselas con las situaciones y dominarlas.

A) Que el plan de acción se malogre (fracaso de la acción)

B) Que el acuerdo fracase (disentimiento o mal entendido)

(Habermas por un momento se ha dedicado a estudiar las miradas de su sparring, y ahora sabe por donde iniciar su argumentación)

El profesor frankfurtiano no desechará lo examinado, sino lo integrará como complemento de su teoría de la acción comunicativa por medio de tres momentos:
FenOMENOLOGÍA

Distingue entre la interpretación de una situación y la ejecución de un plan de acción de esa situación.

Es necesario entender el mundo de la vida para actuar y obrar en él, a través de la interpretación; misma que motivará el desarrollo de planes de acción.

Aquí las situaciones en la práctica se ven limitadas en su interpretación, pues aquellas sólo son definidas en un grado necesario para dominarlas. Las interpretaciones estarán basadas en un acervo de saber dado por la experiencia que fue útil en otro tiempo.

Este análisis del mundo de la vida (en términos de estructura) se propone clarificar la articulación espacio-temporal y social, como es evidente líneas arriba.

Hay una conciencia egológica (Husserl), en la que las estructuras generales de un mundo de la 
A) El mundo de la vida está dado aproblemáticamente (ya vimos por qué)

B) La validez de un mundo intersubjetivamente compartido (no hay mundos privados)

C) Las situaciones son cambiantes, pero no asi los limites del mundo de la vida (que está dado ya siempre) vida, son entendidas como condiciones subjetivas que se apoyan en la experiencia de un mundo de la vida históricamente determinada (esto no plantea cuestiones específicas concretas y variables, sólo generales)

Por otro lado, hay una concepción de sujetividad que constituye al mundo de la vida como marco trascendental de toda experiencia cotidiana posible. Concepción que ha sido útil para la psicología y la sociología (teoría de los sistemas) y que deja fuera a la intersubjetividad.

Así es como para Schütz y Luckmann, las estructuras, de un mundo de la vida están basadas en las vivencias subjetivas de un actor solitario

Habermas apenas da inicio a la contienda, pues inmediatamente después de lo anterior dirá que al apoyarse en las investigaciones fenomenológicas hay un límite conceptual que percibe al mundo de la vida en términos culturalistas, en el que las situaciones para las iniciativas de acción le salen al paso a los actores, a diferencia de lo ya visto en el que el mundo de la vida actuaba a espaldas de aquellos. Y aquí es donde Durkheim cobra relevancia para Habermas, con su concepto de conciencia colectiva, pues este señalará que si los acuerdos se logran, exclusivamente por los esquemas de interpretación en un mundo de la vida, se corre el riesgo de perder el poder de prejuzgar la práctica comunicativa.

Hasta aquí lo dicho en el punto 2, creemos haber mencionado lo fundamental, pero como siempre, a falta de tiempo y espacio, remitimos al lector a los pormenores de este round de las páginas 179 a 193, del texto en cuestión.

El concepto de mundo de la vida, planteado en teoría de la acción comunicativa, sigue en el mismo plano que el concepto trascendental que la fenomenología propone aunque con matices. Nuestro autor examinará el concepto cotidiano del mundo de la vida para acercarse más a las "historias verdaderas". Para ello dirá que en las prácticas comunicativas los actores no sólo se encuentran ante una situación como participantes, sino que se encuentran, también, en exposiciones narrativas que acontecen en un mundo de la vida. En estas exposiciones hay un saber no teórico, sino profano del mundo, que se pretende más acerca de la realidad. Lo narrativo (o hechos teóricos) no sólo cubre necesidades triviales de acuerdo, sino que sirve, además, para "objetivar" la pertenencia a un mundo de la vida de una comunidad, cuyos miembros identificarán a sus propias acciones como algo susceptible de ser narrado, en tanto que en ello les 
va su pertenencia a grupos sociales. "Los colectivos sólo mantienen su identidad en la medida en que las representaciones que de su mundo de vida se ha en sus miembros se solapan suficientemente, condensándose en convicciones de fondo de carácter aproblemático" (Habermas 2001, p. 194).

Pero en "acuerdo" con el profesor alemán, creemos que, efectivamente, la narración sólo nos proporciona una perspectiva en la que situamos el mundo de la vida como sistema cognitivo de referencia dado por el narrar mismo, y en el que se exponen sucesos que en él acaecen, pero que no cubren la totalidad de dicho mundo de la vida. En otras palabras: las narraciones (la historia misma) se refieren a la conservación y reproducción de un mundo de la vida que, en tanto concepto cotidiano de éste, presupone una finalidad cognitiva (el análisis gramatical, al que refiere toda narración, a lo sumo nos permitirá ver cómo describimos, entrelazamos y secuencializamos en un espacio social y en un tiempo histórico las acciones de los individuos, las hazañas de los colectivos y sus destinos, orientados al dominio de situaciones).

Ahora bien, Habermas desplazará lo anterior, es decir, la perspectiva del narrador, hacia la perspectiva de los participantes, en tanto que éstos deben poner en juego el reconocimiento intersubjetivo de pretensiones de validez susceptibles de crítica. Y de ahí pasará a una exposición teórica destinada a explicar la reproducción misma del mundo de la vida a través de estructuras de comunicación.

Comenzará distinguiendo tres aspectos dentro de la acción comunicativa:

A) Funcional de entendimiento (sirve a la tradición y a la renovación del saber cultural)

B) Coordinación de la acción (sirve a la integración social y a la creación de solidaridad)

C) Socialización (sirve a la formación de identidades personales)

Estos aspectos estarán unidos respectivamente a los componentes estructurales de esta forma:

A) Reproducción cultural === Cultura

(Continuación del saber cultural válido)

B) Integración social $===$ Sociedad (Solidaridad de los grupos)

C) Socialización $===$ Personalid ad (Actores capaces de responder de sus acciones) 
A su vez, los componentes del lado derecho, serán entendidos como:

A) Cultura: "acervo de saber, en que los participantes en la comunicación se abastecen de interpretaciones para entenderse sobre algo en el mundo".

B) Sociedad: "ordenaciones legítimas a través de las cuales los participantes en la interacción regulan sus pertenencias a grupos sociales, asegurando con ello la solidaridad".

C) Personalidad: "las competencias que convierten a un sujeto en capaz de lenguajes y de acción, esto es, que lo capaciten para tomar parte en procesos de acuerdo y para afirmar en ellos su propia identidad" (Habermas 2001, p. 196).

Estas estructuras simbólicas del mundo de la vida tejen las interacciones que hacen posible la práctica comunicativa cotidiana. Lo que hace Habermas en este punto es un repaso escueto por algunos autores y su definición del mundo de la vida. Durkheim dirá que el mundo de la vida es la integración social; Parsons piensa que el elemento nuclear de la sociedad está formando grupos socialmente integrados a través de relaciones interpersonales fijadas por el status; Mead, vincula el concepto de mundo de la vida a la mera Socialización de los individuos; los representantes del interaccionismo simbólico (Blumer, Rose, Turner) otorgan excesiva importancia al rol, que deviene en psicología social.

Entramos, en este apartado, a la capacidad habermasiana de esquematización (necesaria, pero no suficiente; por ejemplo: necesaria para clarificar la exposición de ideas e insuficiente porque olvida los aspectos "materiales" (entiéndase corporales) que repercuten seriamente en los sensorios mortales: el hambre, la explotación, la miseria, la contaminación y otras racionalidades no-occidentales, etc.)

Siguiendo la ruta trazada en el apartado anterior, se plantea la siguiente cuestión: ¿qué aportan los distintos procesos de reproducción al mantenimiento de los componentes estructurales (cultura, sociedad, personalidad) del mundo de la vida?

Bien, la cultura (saber válido) aporta a lo social legitimaciones para las instituciones existentes; $\mathrm{y}$ a la personalidad, patrones de comportamiento eficaces.

La sociedad (necesidad de coordinación) aporta a la personalidad, la pertenencia de los individuos o grupos legítimamente regulados; y a la cultura, vinculaciones de carácter moral u obligaciones.

La personalidad (identidad) aporta a la cultura interpretaciones que los individuos producen y a la sociedad, motivaciones para actuar conforme normas. 
Componentes

ESTRUCTURALES

Procesos DE

REPRODUCCIÓN

Cultura

SOCIEDAD

Personalidad

\begin{tabular}{llll}
\hline $\begin{array}{l}\text { Reproducción } \\
\text { cultural }\end{array}$ & $\begin{array}{l}\text { Esquemas de } \\
\text { interpretación } \\
\text { susceptibles de } \\
\text { consenso("saber } \\
\text { válido") }\end{array}$ & Legitimaciones & $\begin{array}{l}\text { Patrones de } \\
\text { comportamiento } \\
\text { eficaces en el } \\
\text { proceso de } \\
\text { formación metas } \\
\text { educativas }\end{array}$ \\
\hline Integración Social & Obligaciones & $\begin{array}{l}\text { Relaciones } \\
\text { interpersonales } \\
\text { legítimamente } \\
\text { reguladas }\end{array}$ & $\begin{array}{l}\text { Pertenencias a } \\
\text { grupos }\end{array}$ \\
\hline Socialización & Interpretaciones & $\begin{array}{l}\text { Motivaciones } \\
\text { para actuar de } \\
\text { conformidad con } \\
\text { las normas }\end{array}$ & $\begin{array}{l}\text { Capacidades } \\
\text { de Interacción } \\
\text { (identidad } \\
\text { personal) }\end{array}$ \\
& & & Pad
\end{tabular}

Fuente: Habermas 2001, p. 202

Ahora Habermas ofrece un contrapeso que evalúe los procesos de reproducción de los componentes estructurales, a través de las siguientes dimensiones:

- Para la cultura $===$ La racionalidad del saber.

- Para la sociedad $===$ La solidaridad de sus miembros.

- Para la personalidad === La autonomía para responder sus acciones.

Asimismo, estas diferenciaciones estructurales tienen perturbaciones que repercuten en: la cultura como pérdida del sentido, en la sociedad como anomía; y en la personalidad como psicopatología. 


\begin{tabular}{|c|c|c|c|c|}
\hline \multicolumn{5}{|l|}{ Componentes } \\
\hline ESTRUCTURALES & & & & \\
\hline $\begin{array}{l}\text { PROCESOS DE } \\
\text { REPRODUCCIÓN }\end{array}$ & Cultura & Sociedad & Personalidad & $\begin{array}{l}\text { DIMENSIÓN } \\
\text { DE EVALUACIÓN }\end{array}$ \\
\hline $\begin{array}{l}\text { Reproducción } \\
\text { cultural }\end{array}$ & $\begin{array}{l}\text { Pérdida de } \\
\text { sentido }\end{array}$ & $\begin{array}{l}\text { Pérdida de } \\
\text { legitimación }\end{array}$ & $\begin{array}{l}\text { Crisis de } \\
\text { orientación y } \\
\text { crisis educativa }\end{array}$ & $\begin{array}{l}\text { Racionalidad } \\
\text { del saber }\end{array}$ \\
\hline $\begin{array}{l}\text { Integración } \\
\text { Social }\end{array}$ & $\begin{array}{l}\text { Inseguridad y } \\
\text { perturbaciones } \\
\text { de la identidad } \\
\text { colectiva }\end{array}$ & Anomía & Alienación & $\begin{array}{l}\text { Solidaridad de } \\
\text { los miembros }\end{array}$ \\
\hline Socialización & $\begin{array}{l}\text { Ruptura de } \\
\text { tradiciones }\end{array}$ & $\begin{array}{l}\text { Pérdida de } \\
\text { motivaciones }\end{array}$ & Psicopatologías & $\begin{array}{l}\text { Autonomía de } \\
\text { la persona }\end{array}$ \\
\hline
\end{tabular}

Fuente: Habermas 2001, p. 203

El asunto de los esquemas no ha acabado, a partir del esquema anterior nuestro autor especificará las funciones que la acción orientada al acuerdo cumple en la reproducción del mundo de la vida:

Componentes

ESTRUCTURALES

Procesos DE

REPRODUCCIÓN

$\begin{array}{lll}\text { Cultura Sociedad } & \text { Personalidad }\end{array}$

\begin{tabular}{llll}
\hline $\begin{array}{l}\text { Reproducción } \\
\text { cultural }\end{array}$ & $\begin{array}{l}\text { Tradición crítica, } \\
\text { adquisición de } \\
\text { saber cultural }\end{array}$ & $\begin{array}{l}\text { Renovación del } \\
\text { saber legitimatorio }\end{array}$ & $\begin{array}{l}\text { Reproducción del } \\
\text { saber eficaz en } \\
\text { los procesos de } \\
\text { formación }\end{array}$ \\
\hline
\end{tabular}

\begin{tabular}{llll}
\hline Integración Social & $\begin{array}{l}\text { Inmunización } \\
\text { de un núcleo de } \\
\text { orientaciones } \\
\text { valorativas }\end{array}$ & $\begin{array}{l}\text { Coordinación de } \\
\text { las acciones a través } \\
\text { del reconocimiento } \\
\text { de pretensiones de } \\
\text { validez }\end{array}$ & $\begin{array}{l}\text { Reproducción de } \\
\text { los patrones de } \\
\text { pertenencia social }\end{array}$ \\
\hline Socialización & Enculturación & $\begin{array}{l}\text { Internalización de } \\
\text { valores }\end{array}$ & $\begin{array}{l}\text { Formación de } \\
\text { la identidad } \\
\text { individual }\end{array}$ \\
\hline
\end{tabular}

Fuente: Habermas 2001, p. 204 
Habermas, ya lo habíamos dicho, sabe lo que hace y lo dice con claridad de autoconciencia (¿y de autocrítica?): "Con determinaciones tan esquemáticamente resumidas como son las expuestas, el concepto de mundo de la vida desarrollado en términos de teoría de la comunicación, todavía está lejos del grado de explicación de su oponente fenomenológico. Con todo, voy a darme por satisfecho con este esbozo..." (Habermas, 2001)

La introducción del concepto mundo de la vida, a través de la teoría de la acción comunicativa, desborda el de la fenomenología, en tanto que puede ser planteado como universal (a toda cultura y época), tal como el concepto de acción comunicativa. Y al igual que en ésta, la autoridad del mejor argumento se impone, y esto, en tanto que los componentes estructurales del mundo de la vida y los procesos que contribuyen a su mantenimiento se diferencian para someterse a los contextos de interacción racionalmente motivados. Un mundo de la vida racionalizado en donde los conflictos no quedan ocultos tras las convicciones que no soportan el discurso argumentado.

Para este punto, Habermas había propuesto entender simultáneamente a la sociedad como sistema y como mundo de la vida, pero lo hace sólo muy brevemente: "Voy a posponer este problema fundamental de toda teoría de la sociedad, es decir, el problema de cómo articular de forma satisfactoria esas dos estrategias conceptuales caracterizadas por las expresiones "sistema" y "mundo de la vida..." (Habermas, 2001, p. 215). Y ofrece un concepto provisional de sociedad entendida "como un sistema que tiene que cumplir las condiciones de mantenimiento propias de los mundos socioculturales de la vida" (ibid)

La sociedad deberá ser entendida como una entidad que al ir evolucionando se diferencia como sistema y como mundo de la vida. La evolución sistémica se determinará por la capacidad de mayor control, mientras que la separación de cultura, sociedad y personalidad, representa la evolución del mundo de la vida, cuyas estructuras son simbólicas.

La propuesta del profesor alemán, tratará de dar razón de la diferencia entre sistema (perspectiva externa en la que la complejidad va en aumento) y mundo de la vida (perspectiva interna en la que la racionalidad debe ir en aumento). Sin embargo, la diferencia no es excluyente, sino, digámoslo así, diferencialmente integrada, y a veces, integrada pese a las diferencias. Cabe hacer notar que el concepto de sociedad, Habermas ya lo había subsumido en el mundo de la vida a través de los componentes estructurales, pero aquí lo está enfocando desde teoría de los sistemas. 
La racionalización del mundo de la vida, aumenta la complejidad de los sistemas, hasta un extremo tal que resultan insuficientes los propios imperativos sistémicos y se ven incapacitados para absorber el mundo de la vida, el cual, paradójicamente había sido instrumentalizado por aquellos: ¿ironía histórica? No lo sabemos hasta ese momento de la obra en cuestión.

\section{CUESTIONAMIENTOS A MANERA DE CONCLUSIÓN PROVISIONAL}

1. La racionalización del mundo de la vida, conlleva en las sociedades un síndrome patológico, digamos destructivo ¿Cuál es el proyecto de racionalidad que hará posible un mundo de la vida planetario de acuerdos etno-intersubjetivos? Si es evidente, en un mundo de la vida cualquiera (incluido el sistema mismo), que la racionalización $\mathrm{NO}$ es discursiva-argumentativa, sino impositiva-instrumental.

2. Habermas ha enunciado que un acercamiento materialista a la reproducción simbólica de un mundo de la vida, requiere de otra plataforma, de otra estrategia, que no identifique a éste con la sociedad uniformizada, ni que la reduzca a elementos simbólicos. Pensamos que esa plataforma deberá ser tan amplia que abarque, prácticamente, múltiples culturas, para lo que el proyecto de racionalidad (como tal) tendrá que ser puesto en entredicho.

3. Los estudios de la Comunicación ¿̇en qué medida se benefician del tipo de discusiones como la arriba expuesta?

\section{BiBLIOGRAFÍA}

Bernstein, R. (1991) Perfiles filosóficos, México, Siglo XXI.

Habermas, J. (1989) El discurso filosófico de la modernidad, Bs.As. Taurus.

Habermas, J. (2001) Teoría de la Acción Comunicativa (2 tomos), Madrid, Taurus. Habermas, J. (2002) Verdad y Justificación, Madrid, Trotta.

Nudler, O. et al.(1996) La racionalidad: su poder y sus límites, Bs. As., Paidós. 